Pacific

Journal of

Mathematics

A GEOMETRIC APPROACH TO THE TOPOLOGICAL DISK THEOREM OF REIFENBERG

GUANGHAO Hong AND LiHE WANG

Volume $233 \quad$ No. 2

December 2007 


\title{
A GEOMETRIC APPROACH TO THE TOPOLOGICAL DISK THEOREM OF REIFENBERG
}

\author{
GuAnghaO Hong AND LiHe WANG
}

\begin{abstract}
We offer a new approach to the classical topological disk theorem of Reifenberg in the codimension- 1 case. Our proof, using $\frac{1}{2}$-level sets of the smoothing of the characteristic function of the domain $\Omega$ as approximating surfaces of $\partial \Omega$, is much simpler than Reifenberg's original one (1960). We also extend the result to parabolic space.
\end{abstract}

\section{Introduction}

We start by recalling the remarkable topological disk theorem in Chapter 4 of the celebrated paper [Reifenberg 1960], which investigated the higher-dimensional Plateau problem.

Definition 1.1 (Reifenberg flat set). We say that a compact set $K \subseteq B_{1}^{n} \subseteq \mathbb{R}^{n}$ is a $m$-dimensional $(\delta, R)$-Reifenberg flat set if for every $a \in K$ and $r \in(0, R]$ with $|a|+r \leq 1$, there exists an $m$-plane $T_{a, r} \in \mathrm{GL}(m, n)$ such that

$$
\mathrm{HD}\left(K \cap B_{r}^{n}(a), T_{a, r} \cap B_{r}^{n}(a)\right) \leq \delta r,
$$

where

$$
\mathrm{HD}(A, B)=\sup \{\operatorname{dist}(a, B): a \in A\}+\sup \{\operatorname{dist}(b, A): b \in B\} .
$$

is the Hausdorff distance.

The definition is only significant for small $\delta>0$.

Theorem 1.2 (Reifenberg's topological disc theorem). If $K$ is Reifenberg flat, then $K \cap B_{\theta}^{n}$ is a $C^{\alpha}$ - topological m-dimensional disk for some $\theta(\delta, R)>0$.

The statement just given is adopted from [Lin and Yang 2002, Chapter 2, p. 58]. Roughly speaking, the theorem shows that if in each ball $B_{r}(a)$ with $a \in K K$ lie in a narrow strip of width less than $2 \delta r$ and moreover it is $\delta r$-dense in the narrow strip, then the surface is locally Euclidean, more precisely, it is locally a bi-Hölder image of the unit ball in $\mathbb{R}^{m}$. It should be noted that the strip is allowed to vary

MSC2000: 49Q15, 28A15.

Keywords: topological disk, Reifenberg flat, parabolic distance. 
both with $a$ and $r$. A typical example of Reifenberg flat set is the well-known Von Koch curve, which is a self-similar Jordan curve and a prototypical fractal set. In the last decade, important works related to this kind of set have appeared: see, for instance, [Capogna et al. 2005; David et al. 2001; David and Toro 1999; Kenig and Toro 1997; 1999; Toro 1997].

A complete proof of Theorem 1.2 can be found in Reifenberg's paper (a sketch is given in the introduction) or in [Morrey 1966, Chapter 10, p. 439]. In Reifenberg's own words, the proof consists of constructing inductively a series of disks which converge to a piece of the surface and are such that each disk is the image of the previous one in such a manner that both the mapping function and its inverse are Lipschitz with constant $k$ where $k$ is fixed throughout the series. Once this series has been constructed the rest is easy. Simply speaking, he constructed the approximate sets locally (mainly due to that the Reifenberg flat condition is a local property), the process involves several averaging processes, each of which introduces complicated estimations, so the proof is in parts unavoidably messy. However, in the codimension- 1 case, we can construct the approximate sets globally, and the estimations are also straightforward. This makes us capable of writing down a direct proof to this theorem.

In the codimension- 1 case, the surface can be seen as the boundary of some domain which we called the Reifenberg flat domain. We will proof this fact by a locally separating theorem in Section 4 of this paper.

Definition 1.3 (Reifenberg flat domain). We say that a domain $\Omega \subset \mathbb{R}^{n}$ is $(\delta, R)$ Reifenberg flat if for every $x \in \partial \Omega$ and $r \in(0, R]$, there exists a unit vector $\vec{n}(x, r) \in$ $\mathbb{R}^{n}$, such that

$$
\begin{aligned}
B_{r}(x) \cap\left\{y \in \mathbb{R}^{n}:\langle y-(x-r \delta \vec{n}\right. & (x, r)), \vec{n}(x, r)\rangle<0\} \\
& \subset B_{r}(x) \cap \Omega \\
& \subset\left\{y \in \mathbb{R}^{n}:\langle y-(x+r \delta \vec{n}(x, r)), \vec{n}(x, r)\rangle<0\right\},
\end{aligned}
$$

where $\langle\cdot, \cdot\rangle$ denotes the inner product in $\mathbb{R}^{n}$.

This kind of domain is geometrically invariant with respect to scaling. The study of elliptic partial differential equations on this domain is interesting [Byun and Wang 2004]. In the first part of this paper, we prove the following theorem.

Theorem 1.4. Let $\Omega \subset \mathbb{R}^{n}$ be a $(\delta, R)$-Reifenberg flat domain such that $\partial \Omega$ contains the origin $O$, and suppose that

$$
B_{2} \cap\left\{x \in \mathbb{R}^{n}: x_{n}<-\delta\right\} \subset B_{2} \cap \Omega \subset\left\{x \in \mathbb{R}^{n}: x_{n}<\delta\right\}
$$

if $\delta>0$ is sufficiently small. Then there exist a map

$$
f: B_{1 / 2} \cap\left\{x_{n}=0\right\} \rightarrow \partial \Omega \cap B_{1}
$$


and numbers $0<\alpha<1$ and $\beta>1$ such that

$$
f\left(B_{1 / 2} \cap\left\{x_{n}=0\right\}\right) \supset \partial \Omega \cap B_{1 / 4}
$$

and

$$
c_{2}|x-y|^{\beta} \leq|f(x)-f(y)| \leq c_{1}|x-y|^{\alpha}
$$

for any $x, y \in B_{1 / 2} \cap\left\{x_{n}=0\right\}$.

The base of our proof is the global construction of the approximating surfaces. We mollify the characteristic function of $\Omega$ and use the $\frac{1}{2}$-level set of the mollified function as the approximate set of $\partial \Omega$. Varying $\varepsilon$, the mollifier scale, we get a series of approximating surfaces which converge to $\partial \Omega$ as $\varepsilon$ tends to zero. There exists a bi-Lipschitz mapping (normal projection operator of a tubular neighborhood) from each approximate set to the next one, and the Lipschitz constant is less than 2 (this estimation need some basic computation). Composing these bi-Lipschitz mappings, we will obtain a bi-Hölder mapping from the $(n-1)$-disk to $\partial \Omega$.

Our method is applicable to some degenerate metric spaces instead of the standard $\mathbb{R}^{n}$ : for instance, the parabolic space $\mathbb{R}^{n+1}$. Section 5 treats the parabolic case. We prove that the boundary of a parabolic Reifenberg flat domain is locally a $C^{\alpha}$-topological disk (Theorem 5.3).

Our construction yields an approximation of Reifenberg flat domains by $C^{2}$ Reifenberg flat domains, both elliptic and parabolic. This type of approximations allows to extend boundary regularity results for solutions of elliptic and parabolic equations to nonsmooth domains.

\section{Approximating surfaces and bi-Lipschitz maps}

Mollifier. Define

$$
\eta(x)=:\left\{\begin{array}{cl}
C_{1}\left(1-|x|^{2}\right)^{3} & \text { if }|x| \leq 1 \\
0 & \text { else }
\end{array}\right.
$$

where the constant $C_{1}$ is adjusted so $\int_{\mathbb{R}^{n}} \eta(x) d x=1$. Next define

$$
\eta_{\varepsilon}(x)=: \varepsilon^{-n} \eta\left(\varepsilon^{-1} x\right) .
$$

Thus

$$
\eta_{\varepsilon}(x) \in C_{0}^{2}\left(B_{2 \varepsilon}\right),
$$

and

$$
\int_{\mathbb{R}^{n}} \eta_{\varepsilon}(x) d x=1 .
$$


Level sets. From now on, we will assume $\Omega$ is as in Theorem 1.4. We define

$$
\begin{gathered}
\chi_{\Omega}(x):= \begin{cases}1 & \text { if } x \in \Omega, \\
0 & \text { otherwise, }\end{cases} \\
\chi_{\varepsilon}(x):=\chi_{\Omega} * \eta_{\varepsilon}, \quad L_{\varepsilon}:=\left\{x \in \mathbb{R}^{n}: \chi_{\varepsilon}(x)=\frac{1}{2}\right\} .
\end{gathered}
$$

Curvature estimate of the $\frac{1}{2}$-level set $\boldsymbol{L}_{\varepsilon}$. We make some derivative estimates for $\chi_{\varepsilon}$ near $L_{\varepsilon}$. Fix $x \in L_{\varepsilon}$ and let $y \in \partial \Omega$, s.t. $d=:|y-x|=\inf _{z \in \partial \Omega}|z-x|$. Obviously $d<\varepsilon$. In fact, if $\varepsilon \leq d$, then $B_{\varepsilon}(x) \in \Omega$ or $B_{\varepsilon}(x) \in \bar{\Omega}^{c}$, that implies $\chi_{\varepsilon}(x)=1$ or $\chi_{\varepsilon}(x)=0$. Set

$$
\begin{aligned}
A_{1} & :=\{z:\langle z-(y-4 \varepsilon \delta \vec{n}(y, 4 \varepsilon)), \vec{n}(y, 4 \varepsilon)\rangle<0\} \cap B_{4 \varepsilon}(y), \\
A_{2} & :=\{z:\langle z-(y+4 \varepsilon \delta \vec{n}(y, 4 \varepsilon)), \vec{n}(y, 4 \varepsilon)\rangle \geq 0\} \cap B_{4 \varepsilon}(y)-A_{1}, \\
A_{3} & :=B_{4 \varepsilon}(y)-\left(A_{1} \cup A_{2}\right), \\
T & :=\partial A_{1}-\partial B_{4 \varepsilon}(y) .
\end{aligned}
$$

Lemma 2.1. $x \in A_{2}$ and $d \leq 8 \varepsilon \delta$.

Proof. If $x \in A_{1}$, since $d<\varepsilon$,

$$
\chi_{\varepsilon}(x)=\int_{B_{\varepsilon}(x) \cap \Omega} \eta_{\varepsilon}(z-x) d z \geq \int_{B_{\varepsilon}(x) \cap A_{1}} \eta_{\varepsilon}(z-x) d z>\frac{1}{2} .
$$

Similarly, if $x \in A_{3}$, then $\chi_{\varepsilon}(x)<\frac{1}{2}$. Thus $x \in A_{2}$.

$B_{8 \varepsilon \delta}(x) \cap \partial \Omega \neq \varnothing$ implies $d \leq 8 \varepsilon \delta$.

From now on, we will assume that $\vec{n}(y, 4 \varepsilon)=-e_{n}=(0, \ldots, 0,-1)$. Let $\hat{e}$ be any unit vector in $\mathbb{R}^{n-1}$ and define $e:=(\hat{e}, 0) \in \mathbb{R}^{n}$, so $\left\langle e, e_{n}\right\rangle=0$.

Lemma 2.2. For any $\bar{x} \in A_{2} \cap B_{\varepsilon}(y)$, we have

$$
\begin{gathered}
\frac{\partial}{\partial e_{n}} \chi_{\varepsilon}(\bar{x}) \geq C_{2} \varepsilon^{-1}, \quad\left|\frac{\partial}{\partial e} \chi_{\varepsilon}(\bar{x})\right| \leq C_{3} \delta \varepsilon^{-1}, \\
\left|\frac{\partial^{2}}{\partial e^{2}} \chi_{\varepsilon}(\bar{x})\right| \leq C_{4} \delta \varepsilon^{-2}, \quad\left|\frac{\partial^{2}}{\partial e \partial e_{n}} \chi_{\varepsilon}(\bar{x})\right| \leq C_{4} \delta \varepsilon^{-2}, \quad\left|\frac{\partial^{2}}{\partial e_{n}^{2}} \chi_{\varepsilon}(\bar{x})\right| \leq C_{5} \varepsilon^{-2} .
\end{gathered}
$$

Proof.

$$
\begin{aligned}
\frac{\partial}{\partial e_{n}} \chi_{\varepsilon}(\bar{x}) & =\left(\chi_{\Omega} * \frac{\partial}{\partial e_{n}} \eta_{\varepsilon}\right)(\bar{x})=\int_{\Omega} \frac{\partial}{\partial e_{n}} \eta_{\varepsilon}(\bar{x}-z) d z \\
& =\int_{A_{1} \cap B_{\varepsilon}(\bar{x})} \frac{\partial}{\partial e_{n}} \eta_{\varepsilon}(\bar{x}-z) d z+\int_{\Omega \cap A_{2} \cap B_{\varepsilon}(\bar{x})} \frac{\partial}{\partial e_{n}} \eta_{\varepsilon}(\bar{x}-z) d z=: I_{1}+I_{2} .
\end{aligned}
$$


By Green's Formula,

$$
\begin{aligned}
I_{1} & =\int_{T \cap B_{\varepsilon}(\bar{x})} \eta_{\varepsilon}(\bar{x}-s) d s \geq \int_{T \cap B_{\varepsilon / 2}(\bar{x})} \eta_{\varepsilon}(\bar{x}-s) d s \geq C_{2}^{\prime} \varepsilon^{-n} \cdot C_{2}^{\prime \prime}\left(\frac{\varepsilon}{2}\right)^{n-1} \\
& =C_{2}^{\prime \prime \prime} \varepsilon^{-1}, \\
\left|I_{2}\right| & \leq \int_{A_{2} \cap B_{\varepsilon}(\bar{x})}\left|\frac{\partial}{\partial e_{n}} \eta_{\varepsilon}(\bar{x}-z)\right| d z \leq C_{2}^{(4)} \varepsilon^{-n} \varepsilon^{-1} \cdot C_{2}^{(5)} \delta \varepsilon^{n}=C_{2}^{(6)} \delta \varepsilon^{-1} .
\end{aligned}
$$

If $C_{2}^{(6)} \delta<C_{2}^{\prime \prime \prime} / 2$, set $C_{2}=C_{2}^{\prime \prime \prime} / 2$. We have

$$
\begin{aligned}
\frac{\partial}{\partial e} \chi_{\varepsilon}(\bar{x}) & =\int_{\Omega} \frac{\partial}{\partial e} \eta_{\varepsilon}(\bar{x}-z) d z \\
& =\int_{A_{1} \cap B_{\varepsilon}(\bar{x})} \frac{\partial}{\partial e} \eta_{\varepsilon}(\bar{x}-z) d z+\int_{\Omega \cap A_{2} \cap B_{\varepsilon}(\bar{x})} \frac{\partial}{\partial e} \eta_{\varepsilon}(\bar{x}-z) d z=: I_{3}+I_{4} ;
\end{aligned}
$$

but $I_{3}=0$ by Green's formula, and

$$
\left|I_{4}\right| \leq \int_{A_{2} \cap B_{\varepsilon}(\bar{x})}\left|\frac{\partial}{\partial e} \eta_{\varepsilon}(\bar{x}-z)\right| d z \leq C_{2}^{(6)} \delta \varepsilon^{-1},
$$

like $I_{2}$. Set $C_{3}=C_{2}^{(6)}$. We have

$\frac{\partial^{2}}{\partial e^{2}} \chi_{\varepsilon}(\bar{x})=\int_{A_{1} \cap B_{\varepsilon}(\bar{x})} \frac{\partial^{2}}{\partial e^{2}} \eta_{\varepsilon}(\bar{x}-z) d z+\int_{\Omega \cap A_{2} \cap B_{\varepsilon}(\bar{x})} \frac{\partial^{2}}{\partial e^{2}} \eta_{\varepsilon}(\bar{x}-z) d z=: I_{5}+I_{6} ;$

now $I_{5}=0$ by Green's Formula, and

$$
\left|I_{6}\right| \leq \int_{A_{2} \cap B_{\varepsilon}(\bar{x})}\left|\frac{\partial^{2}}{\partial e^{2}} \eta_{\varepsilon}(\bar{x}-z)\right| d z \leq C_{4}^{\prime} \varepsilon^{-n} \varepsilon^{-2} \cdot C_{2}^{(5)} \delta \varepsilon^{n}=C_{4} \delta \varepsilon^{-2} .
$$

We omit the proofs of the last two inequalities, which are similar to ones above.

Since $\nabla \chi_{\varepsilon}(x) \neq 0$ for $x \in L_{\varepsilon} \cap B_{1}, L_{\varepsilon} \cap B_{1}$ is a $C^{2}(n-1)$-dimensional manifold and can be represented as the graph of some function from $\mathbb{R}^{n-1}$ to $\mathbb{R}$ locally by the Implicit Function Theorem. If you take a point $\hat{x} \in L_{\varepsilon} \cap B_{1}$, and $\hat{y}$ is the nearest point from $\partial \Omega$ to $\hat{x}$, then in $A_{2} \cap B_{\varepsilon}(\hat{y})$, the function can be taken as

$$
x_{n}=\varphi\left(x_{1}, \ldots, x_{n-1}\right)=: \varphi\left(x_{T}\right) .
$$

Lemma 2.3.

$$
\left|\frac{\partial}{\partial \hat{e}} \varphi\left(x_{T}\right)\right|<\frac{C_{3}}{C_{2}} \delta \quad \text { and } \quad\left|\frac{\partial^{2}}{\partial \hat{e}^{2}} \varphi\left(x_{T}\right)\right| \leq C_{6} \delta \varepsilon^{-1} .
$$

Proof. By the Implicit Function Theorem, we have

$$
\frac{\partial}{\partial \hat{e}} \varphi\left(x_{T}\right)=-\frac{\frac{\partial}{\partial e} \chi_{\varepsilon}\left(x_{T}, \varphi\left(x_{T}\right)\right)}{\frac{\partial}{\partial e_{n}} \chi_{\varepsilon}\left(x_{T}, \varphi\left(x_{T}\right)\right)},
$$


so $\left|\frac{\partial}{\partial \hat{e}} \varphi\left(x_{T}\right)\right|<\frac{C_{3}}{C_{2}} \delta ;$ and

$\frac{\partial^{2}}{\partial \hat{e}^{2}} \varphi\left(x_{T}\right)=-\frac{\frac{\partial^{2}}{\partial e^{2}} \chi_{\varepsilon}\left(x_{T}, \varphi\left(x_{T}\right)\right)+\frac{\partial^{2}}{\partial e_{n}^{2}} \chi_{\varepsilon} \cdot\left(\frac{\partial}{\partial \hat{e}} \varphi\left(x_{T}\right)\right)^{2}+2 \frac{\partial^{2}}{\partial e \partial e_{n}} \chi_{\varepsilon} \cdot\left(\frac{\partial}{\partial \hat{e}} \varphi\left(x_{T}\right)\right)}{\frac{\partial}{\partial e_{n}} \chi_{\varepsilon}\left(x_{T}, \varphi\left(x_{T}\right)\right)}$,

so

$\left|\frac{\partial^{2}}{\partial \hat{e}^{2}} \varphi\left(x_{T}\right)\right| \leq \frac{C_{4} \delta \varepsilon^{-2}+C_{5} \varepsilon^{-2}\left(\frac{C_{3}}{C_{2}}\right)^{2} \delta^{2}+2 C_{4} \delta \varepsilon^{-2} \frac{C_{3}}{C_{2}} \delta}{C_{2} \varepsilon^{-1}} \leq \frac{2 C_{4} \delta \varepsilon^{-2}}{C_{2} \varepsilon^{-1}}=C_{6} \delta \varepsilon^{-1}$,

where the second inequality holds for $\delta$ small enough.

Corollary 2.4. The principal curvature of $L_{\varepsilon}$ in any tangent direction is less than $C_{6} \delta \varepsilon^{-1}$. If $C_{6} \delta<1$, a tubular neighborhood with radii of $\varepsilon$ exists.

Closeness of $\boldsymbol{L}_{\boldsymbol{\varepsilon}}$ to $\partial \boldsymbol{\Omega}$. If $E$ is an (n-1)-dimensional manifold in $\mathbb{R}^{n}$ having a tubular neighborhood with radii of $\tau$, we denote by $N(E, s)$ an $s$-tubular neighborhood of $E$ for $0<s \leq \tau$, and by $\pi$ the normal projection map of the tubular neighborhood.

Lemma 2.5. $\partial \Omega \cap B_{r-\varepsilon} \subset N\left(L_{\varepsilon} \cap B_{r}, 4 \varepsilon \delta\right)$ for $r \leq 1$.

Proof. Given any $\bar{x} \in \partial \Omega \cap B_{r-\varepsilon}$, we have

$$
\begin{aligned}
B_{4 \varepsilon}(\bar{x}) \cap\{x:\langle x-(\bar{x}-4 \varepsilon \delta \vec{n}(\bar{x}, 4 \varepsilon)) & , \vec{n}(\bar{x}, 4 \varepsilon)\rangle<0\} \\
& \subset B_{4 \varepsilon}(\bar{x}) \cap \Omega \\
& \subset\{x:\langle x-(\bar{x}+4 \varepsilon \delta \vec{n}(\bar{x}, 4 \varepsilon)), \vec{n}(\bar{x}, 4 \varepsilon)\rangle<0\} .
\end{aligned}
$$

Thus $\chi_{\varepsilon}(\bar{x}-2 \varepsilon \vec{n})=1, \chi_{\varepsilon}(\bar{x}+2 \varepsilon \vec{n})=0$. By the continuity of $\chi_{\varepsilon}$, there exists $s \in(-1,1)$ such that $\chi_{\varepsilon}(\bar{x}+2 s \varepsilon \vec{n})=\frac{1}{2}$. In fact, $|s|<2 \delta$ by the proof of Lemma 2.1 .

Taking $\varepsilon=4^{-k}, k=1,2, \ldots$, we gain a series of approximating surfaces $L_{4^{-k}}$. Define $\mathscr{Y}_{0}:=\left\{x_{n}=0\right\} \cap B_{1 / 2}$ and $\mathscr{S}_{k}:=L_{4^{-k}} \cap B_{r(k)}$, where $r(k)=\frac{1}{2}+\sum_{j=1}^{k} 4^{-j}$.

Corollary 2.6. $\mathscr{S}_{k} \in N\left(L_{4^{-k+1}}, 6 \cdot 4^{-k+1} \delta\right)$.

Proof. For any $x \in \mathscr{T}_{k}$, there exists $y \in \partial \Omega \cap B_{5 / 6}$ such that $|x-y|<8 \cdot 4^{-k} \delta$, and $z \in L_{4^{-k+1}} \cap B_{1}$ such that $|y-z|<4 \cdot 4^{-k+1} \delta$, so $|x-z|<6 \cdot 4^{-k+1} \delta$.

\section{Bi-Lipschitz maps.}

Lemma 2.7. $\pi: \mathscr{Y}_{k} \rightarrow L_{4^{-k+1}}$ is inject, and if $\delta$ is small enough, given any $z^{1}$, $z^{2} \in \mathscr{Y}_{k}$, satisfying $\left|z^{1}-z^{2}\right| \leq 4^{-k}$, then

$$
\frac{1}{2}\left|\pi\left(z^{1}\right)-\pi\left(z^{2}\right)\right| \leq\left|z^{1}-z^{2}\right| \leq 2\left|\pi\left(z^{1}\right)-\pi\left(z^{2}\right)\right| .
$$


Proof. We write $x^{1}=: \pi\left(z^{1}\right), x^{2}=: \pi\left(z^{2}\right) \in L_{4^{-k+1}}$. For simplicity, we will still write $4^{-k+1}$ as $\varepsilon$ sometimes. By choosing an appropriate coordinate system, we can assume that original point $O$ is the nearest point of $\partial \Omega$ to $z^{1}$, and $\vec{n}(0,4 \varepsilon)=-e_{n}$. Define

$$
\begin{aligned}
& A_{1}:=\left\{x: x_{n}>4 \varepsilon \delta\right\} \cap B_{4 \varepsilon} ; \\
& A_{2}:=\left\{x:-4 \varepsilon \delta \leq x_{n} \leq 4 \varepsilon \delta\right\} \cap B_{4 \varepsilon} ; \\
& A_{3}:=\left\{x: x_{n}<-4 \varepsilon \delta\right\} \cap B_{4 \varepsilon} .
\end{aligned}
$$

Then $A_{1} \subset \Omega, A_{3} \subset \bar{\Omega}^{c}$, and $x^{1}, x^{2}, z^{1}, z^{2} \in A_{2} \cap B_{\varepsilon}$.

We assume $L_{\varepsilon} \cap B_{\varepsilon}$ is the graph of function $x_{n}=\varphi\left(x_{T}\right)=\varphi\left(x_{1}, \ldots, x_{n-1}\right)$ and $L_{\varepsilon / 4} \cap B_{\varepsilon}$ is the graph of function $z_{n}=\psi\left(z_{T}\right)$. Thus we can write

$$
\begin{array}{ll}
x^{1}=\left(x_{T}^{1}, \varphi\left(x_{T}^{1}\right)\right), & x^{2}=\left(x_{T}^{2}, \varphi\left(x_{T}^{2}\right)\right), \\
z^{1}=\left(z_{T}^{1}, \psi\left(z_{T}^{1}\right)\right), & z^{2}=\left(z_{T}^{2}, \psi\left(z_{T}^{2}\right)\right) .
\end{array}
$$

We define $l=:\left|x_{T}^{1}-x_{T}^{2}\right|, \hat{l}=:\left|z_{T}^{1}-z_{T}^{2}\right|$. By assumption,

$$
\hat{l} \leq \varepsilon / 4 \text { and } l \leq \varepsilon / 2 .
$$

By Lemma 2.3 and the Differential Mean Value Theorem,

$$
\left|\varphi\left(x_{T}^{1}\right)-\varphi\left(x_{T}^{2}\right)\right| \leq l \cdot \frac{C_{3}}{C_{2}} \delta, \quad\left|\psi\left(z_{T}^{1}\right)-\psi\left(z_{T}^{2}\right)\right| \leq \hat{l} \cdot \frac{C_{3}}{C_{2}} \delta .
$$

Then

$$
\begin{aligned}
& l \leq\left|x^{1}-x^{2}\right| \leq \sqrt{l^{2}+2\left(\frac{C_{3}}{C_{2}} \delta\right)^{2} l^{2}} \leq \frac{11}{10} l \quad \text { (using } \delta \text { small), } \\
& \hat{l} \leq\left|z^{1}-z^{2}\right| \leq \frac{11}{10} \hat{l} .
\end{aligned}
$$

Since $\pi\left(z^{1}\right)=x^{1}$ and $\pi\left(z^{2}\right)=x^{2}$, there exist $\xi, \zeta \in \mathbb{R}$, such that

$$
z^{1}=x^{1}+\xi v\left(x^{1}\right) \text { and } z^{2}=x^{2}+\zeta v\left(x^{2}\right),
$$

where $v\left(x^{1}\right), v\left(x^{2}\right)$ are inward-pointing unit normal vectors $L_{\varepsilon}$, at $x^{1}, x^{2}$. Explicitly,

$$
\begin{aligned}
& v_{i}\left(x^{1}\right)=\frac{-D_{i} \varphi\left(x_{T}^{1}\right)}{\sqrt{1+\left|\nabla \varphi\left(x_{T}^{1}\right)\right|^{2}}} \text { for } i=1, \ldots, n-1, \\
& v_{n}\left(x^{1}\right)=\frac{1}{\sqrt{1+\left|\nabla \varphi\left(x_{T}^{1}\right)\right|^{2}}},
\end{aligned}
$$

and likewise for $v\left(x^{2}\right)$. By Corollary 2.6 we have

$|\xi|, \quad|\zeta| \leq 6 \varepsilon \delta$ 
moreover, $\left|v_{i}\left(x^{j}\right)\right|<C_{3} / C_{2} \delta$ for $i=1, \ldots, n-1$, and

$$
v_{n}\left(x^{j}\right)>\frac{1}{\sqrt{1+\left(C_{3} / C_{2} \delta\right)^{2}}} \text { for } j=1,2,
$$

by Lemma 2.3. These two inequalities show that $v\left(x^{j}\right)$ is very close to $e_{n}$ as $\delta$ is very small.

Subtracting the second equality in (2-3) from the first, we get

$$
\begin{aligned}
z_{i}^{1}-z_{i}^{2} & =x_{i}^{1}-x_{i}^{2}+\xi\left(v_{i}\left(x^{1}\right)-v_{i}\left(x^{2}\right)\right)+(\xi-\zeta) v_{i}\left(x^{2}\right), \\
z_{n}^{1}-z_{n}^{2} & =x_{n}^{1}-x_{n}^{2}+\xi\left(v_{n}\left(x^{1}\right)-v_{n}\left(x^{2}\right)\right)+(\xi-\zeta) v_{n}\left(x^{2}\right) .
\end{aligned}
$$

By Lemma 2.3 and the Differential Mean Value Theorem, we obtain

$$
\begin{aligned}
\left|v_{i}\left(x^{1}\right)-v_{i}\left(x^{2}\right)\right| & \leq\left|D_{i} \varphi\left(x_{T}^{2}\right)-D_{i} \varphi\left(x_{T}^{1}\right)\right|+\left|\nabla \varphi\left(x_{T}^{1}\right)\right|^{2} \cdot\left|\nabla \varphi\left(x_{T}^{2}\right)-\nabla \varphi\left(x_{T}^{1}\right)\right| \\
& \leq 2 l \cdot C_{6} \delta \varepsilon^{-1},
\end{aligned}
$$

and likewise

$$
\begin{aligned}
\left|v_{n}\left(x^{1}\right)-v_{n}\left(x^{2}\right)\right| & \leq\left(\left|\nabla \varphi\left(x_{T}^{1}\right)\right|+\left|\nabla \varphi\left(x_{T}^{2}\right)\right|\right) \cdot\left|\nabla \varphi\left(x_{T}^{1}\right)-\nabla \varphi\left(x_{T}^{2}\right)\right| \\
& \leq l \cdot C_{6} \delta \varepsilon^{-1} .
\end{aligned}
$$

Equality (2-6) implies

$$
\begin{aligned}
|\xi-\zeta| & \leq \sqrt{1+\left(\frac{C_{3}}{C_{2}} \delta\right)^{2}}\left(\left|z_{n}^{1}-z_{n}^{2}\right|+\left|x_{n}^{1}-x_{n}^{2}\right|+|\xi| \cdot\left|v_{n}\left(x^{1}\right)-v_{n}\left(x^{2}\right)\right|\right) \\
& \leq \sqrt{1+\left(\frac{C_{3}}{C_{2}} \delta\right)^{2}}\left(\hat{l} \cdot \frac{C_{3}}{C_{2}} \delta+l \cdot \frac{C_{3}}{C_{2}} \delta+5 \varepsilon \delta \cdot l \cdot C_{6} \delta \varepsilon^{-1}\right) \leq C_{7} \delta(l+\hat{l}) ;
\end{aligned}
$$

here the second inequality follows using (2-1), (2-4) and (2-7).

Using the triangle inequality, (2-5) implies

$$
\hat{l} \leq l+|\xi| \cdot\left|v_{i}\left(x^{1}\right)-v_{i}\left(x^{2}\right)\right|+|\xi-\zeta| \cdot\left|v_{i}\left(x^{2}\right)\right| \leq\left(1+C_{8} \delta^{2}\right) l+C_{7} \frac{C_{3}}{C_{2}} \delta^{2} \hat{l} .
$$

Thus if $\delta$ is small enough, we obtain $\hat{l} \leq \frac{11}{10} l$. Similarly, one can show that $\hat{l} \geq \frac{9}{10} l$. Combining these two bounds with (2-2), we conclude the proof.

We define $g_{k}: \mathscr{S}_{k-1} \rightarrow \mathscr{Y}_{k}$ satisfying $\pi\left(g_{k}(x)\right)=x$ for $k=1,2, \ldots$, and $h_{k}$ : $\mathscr{S}_{0} \rightarrow \mathscr{S}_{k}$ by setting $h_{k}=: g_{k} \circ \cdots \circ g_{1}$.

Corollary 2.8. $\left|h_{k}(x)-h_{k-1}(x)\right|=\left|g_{k}\left(h_{k-1}(x)\right)-h_{k-1}(x)\right|<6 \cdot 4^{-k+1} \delta$. 


\section{Proof of Theorem 1.4}

Corollary 2.8 implies that $\left\{x, h_{1}(x), \ldots, h_{k}(x), \ldots\right\}$ is a Cauchy sequence. Letting $f(x)$ be the limit point, Lemma 2.1 indicates that $f(x) \in \partial \Omega \cap B_{1}$. Thus we get a map

$$
f: B_{1 / 2} \cap\left\{x_{n}=0\right\}=\mathscr{Y}_{0} \rightarrow \partial \Omega \cap B_{1} .
$$

Given $x$ and $y \in \mathscr{T}_{0}$, define $d:=|f(x)-f(y)|$. If $d<\frac{10}{3} 4^{2} \delta$, choose $k$ satisfying

$$
\frac{1}{3} 4^{-k+2} \delta \leq \frac{1}{10} d<\frac{1}{3} 4^{-k+3} \delta,
$$

then by triangle inequality and Corollary 2.8 ,

$$
\frac{8}{10} d<\left|h_{k}(x)-h_{k}(y)\right|<\frac{12}{10} d .
$$

From Lemma 2.7,

$$
2^{-k}|x-y| \leq\left|h_{k}(x)-h_{k}(y)\right| \leq 2^{k}|x-y| .
$$

However, (3-1) implies

$$
\log _{4} d^{-1}+2+\log _{4} \frac{10}{3}+\log _{4} \delta \leq k<\log _{4} d^{-1}+3+\log _{4} \frac{10}{3}+\log _{4} \delta .
$$

Combining this with (3-2) and (3-3), we obtain

$$
c_{2}|x-y|^{\beta} \leq d \leq c_{1}|x-y|^{\alpha},
$$

where $\alpha=1 /(1+\lambda), \beta=1 /(1-\lambda)$, for $\lambda=\log _{4} 2=\frac{1}{2}$.

Finally, the inclusion $f\left(\mathscr{Y}_{0}\right) \supset \partial \Omega \cap B_{1 / 4}$ follows from Lemma 2.5. This concludes the proof of Theorem 1.4.

Remark 3.1. From the argument it is easy to see that $\alpha$ and $\beta$ can be taken arbitrarily close to 1 as long as $\delta$ is sufficiently small and $c_{1}, c_{2}$ are allowed to be large.

\section{The locally separating theorem}

Theorem 4.1. Let $K$ be a set satisfying Definition 1.1 in the case $m=n-1$. We assume that the original point $O \in K$ and $T_{O, 1}=\left\{x \in \mathbb{R}^{n}: x_{1}=0\right\}$. Set

$$
\begin{aligned}
& A_{1}:=B_{1} \cap\left\{x: x_{1}<-2 \delta\right\}, \\
& A_{2}:=B_{1} \cap\left\{x:-2 \delta \leq x_{1} \leq 2 \delta\right\}, \\
& A_{3}:=B_{1} \cap\left\{x: x_{1}>2 \delta\right\} .
\end{aligned}
$$

(I) The set $B_{3 / 4}-K$ has at least two connected components, one containing $A_{1} \cap B_{3 / 4}$ and the other $A_{3} \cap B_{3 / 4}$. We denote by $\Omega_{1}$ and $\Omega_{2}$, respectively, these two components.

(II) $B_{1 / 2}-K \subset \Omega_{1} \cup \Omega_{2}$. 
(III) For any $a \in K \cap B_{1 / 2}$ and $r \in(0, R]$ satisfying $|a|+r<1 / 2, B_{r}(a) \cap \Omega_{i} \neq \varnothing$ for $i=1,2$.

(IV) $K \cap B_{1 / 2}=\partial \Omega_{1} \cap B_{1 / 2}=\partial \Omega_{2} \cap B_{1 / 2}$.

Proof. We prove the disconnectedness of $B_{3 / 4}-K$ by contradiction. If $B_{3 / 4}-K$ is connected, it is arcwise connected. So we can choose a path which connect $z_{1}=:\left(-\frac{1}{4}, 0, \ldots, 0\right)$ with $z_{2}=:\left(\frac{1}{4}, 0, \ldots, 0\right)$ and is contained in $B_{3 / 4}-K$. We denote it as $\gamma:[0, l] \rightarrow B_{3 / 4}-K$ (arclength parameter $t$ is used), which satisfy $\gamma(0)=z_{1}$ and $\gamma(l)=z_{2}$. Set $d:=\inf _{t \in[0, l]} \operatorname{dist}(\gamma(t), K)$. Any path connecting $z_{1}$ with $z_{2}$ and contained in $B_{1}$ must traverse $T_{O, 1}$. So the distance of the path to $K$ is less than $\delta$. Hence, we assume $d<\delta$ at first. We will move $\gamma$ away from $K$ constantly and enlarge the distance of the path to $k$ till it is bigger than $\delta$. By this way, we will get a contradiction.

Let $t_{1}$ be such that $\gamma\left(t_{1}\right) \in \partial A_{1}$ and $\gamma(t) \in A_{2} \cup A_{3}$, for $t \geq t_{1}$. We may as well assume that $\gamma(t) \in A_{1}$ for $0 \leq t<t_{1}$ (otherwise, we connect $z_{1}$ with $\gamma\left(t_{1}\right)$ by a straight line and take the new path as $\gamma)$. Let $t^{*}$ be such that $\gamma\left(t^{*}\right) \in \partial A_{3}$ and $\gamma(t) \in A_{1} \cup A_{2}$, for $t \leq t^{*}$. For the same reason, we assume $\gamma(t) \in A_{3}$, for $t^{*}<t \leq l$.

Now define $r_{1}:=\operatorname{dist}\left(\gamma\left(t_{1}\right), K\right)$; obviously, $d \leq r_{1}<3 \delta$. Letting $y_{1}$ be a nearest point to $\gamma\left(t_{1}\right)$ from $K$, we consider $T_{y_{1}, 5 r_{1}}$. Because of

$$
\operatorname{dist}\left(\gamma\left(t_{1}\right), y_{1}\right) \leq \operatorname{dist}\left(\gamma\left(t_{1}\right), y\right), \quad \text { for any } y \in K
$$

and

$$
\operatorname{HD}\left(K \cap B_{5 r_{1}}\left(y_{1}\right), T_{y_{1}, 5 r_{1}} \cap B_{5 r_{1}}\left(y_{1}\right)\right)<5 r_{1} \delta,
$$

$\gamma\left(t_{1}\right)-y_{1}$ is almost vertical to $T_{y_{1}, 5 r_{1}}$, more precisely,

$$
\measuredangle\left(\gamma\left(t_{1}\right)-y_{1}, T_{y_{1}, 5 r_{1}}^{\perp}\right)<C(n) \delta .
$$

Hence, if we move $\gamma\left(t_{1}\right)$ to $x_{1}:=\gamma\left(t_{1}\right)+d\left(\gamma\left(t_{1}\right)-y_{1}\right)$, then

$$
\operatorname{dist}\left(x_{1}, K\right)>r_{1}+0.9 d \text { (use the smallness of } \delta \text { ). }
$$

If we have chosen $t_{k}, r_{k}, y_{k}$ and $x_{k}$, then let $t_{k+1}$ be such that

$$
\gamma\left(t_{k+1}\right) \in \partial B_{r_{k} / 3}\left(\gamma\left(t_{k}\right)\right)
$$

satisfying

$$
\gamma(t) \in B_{r_{k} / 3}\left(\gamma\left(t_{k}\right)\right) \text { for } t_{k} \leq t<t_{k+1} .
$$

Obviously, $t_{k+1}-t_{k} \geq r_{k} / 3 \geq d / 3$. Let $r_{k+1}, y_{k+1}$ and $x_{k+1}$ be defined as previous process. Thanks to the lower bound of step length, there exists $j \in N$, such that $t_{j-1}<t^{*} \leq t_{j}$.

Let $\tilde{\gamma}$ be a path consisting of straight line segments $x_{k} x_{k+1}(k=0, \ldots, j)$, with $x_{0}=z_{1}$ and $x_{j+1}=z_{2}$. We claim that $\operatorname{dist}(\tilde{\gamma}, K)>\min \{1.8 d, \delta\}$. In fact, 
The straight lines $z_{1} x_{1}$ and $x_{j} z_{2}$ are contained in $A_{1}$ and $A_{3}$ respectively, so their distances to $K$ are greater than $\delta$. For any straight line $x_{k} x_{k+1}(k=1, \ldots, j-1)$, we assume $\operatorname{dist}\left(x_{k} x_{k+1}, K\right) \leq \delta$. The three relations

$$
\begin{gathered}
\measuredangle\left(\gamma\left(t_{k}\right)-y_{k}, T_{y_{k}, 5 r_{k}}^{\perp}\right)<C(n) \delta, \\
\gamma\left(t_{k+1}\right) \in \partial B_{r_{k} / 3}\left(\gamma\left(t_{k}\right)\right), \\
\operatorname{HD}\left(K \cap B_{5 r_{1}}\left(y_{1}\right), T_{y_{1}, 5 r_{1}} \cap B_{5 r_{1}}\left(y_{1}\right)\right)<5 r_{1} \delta
\end{gathered}
$$

together imply that

$$
\measuredangle\left(\gamma\left(t_{k+1}\right)-y_{k+1}, \gamma\left(t_{k}\right)-y_{k}\right)<C(n) \delta .
$$

Therefore, $\operatorname{dist}\left(x_{k} x_{k+1}, K\right)>\min \left\{r_{k}, r_{k+1}\right\}+0.8 d \geq 1.8 d$.

If $1.8 d<\delta$, we continue to move $\tilde{\gamma}$. After finitely many such moves, we obtain a path whose distance to $K$ exceeds $\delta$. This proves (I).

For any point $b \in A_{2} \cap B_{1 / 2}-K$, define $d_{b}:=\operatorname{dist}(b, K)$. We can move $b$ (away from $K$ ) to $b_{1}$ such that $\operatorname{dist}\left(b_{1}, K\right) \geq 1.9 d_{b}$ by the previous method. If necessary, we continue to move $b_{1}$ to $b_{2}, \ldots$ After finite (say $i$ ) times, it will happen that $\operatorname{dist}\left(b_{i}, K\right)>3 \delta$. So $b_{i} \in A_{1}$ (or $A_{3}$ ). However, the straight lines $b_{k} b_{k+1}(k=0, \ldots, i-1)$, where $b_{0}=b$, are contained in $B_{1}-K$. So $b \in \Omega_{1}$ or $b \in \Omega_{2}$. This proves (II).

For any $a \in K \cap B_{1 / 2}$ and $r \in(0, R]$ satisfying $|a|+r<1 / 2$, let $J \in N$ be such that $2^{J-1} r \leq 4 \delta<2^{J} r$. We consider $B_{2^{k} r}(a)$ and $T_{a, 2^{k} r}$ for $k=0,1, \ldots, J$. For any $k$, the set $B_{2^{k_{r}}}(a)-N\left(T_{a, 2^{k} r}, 2^{k} r \delta\right)$ have just two parts. Because of the closeness of $T_{a, 2^{k} r}$ and $T_{a, 2^{k+1} r}$, the two parts of $B_{2^{k} r}(a)-N\left(T_{a, 2^{k} r}, 2^{k} r \delta\right)$ meet the two parts of $B_{2^{k+1} r}(a)-N\left(T_{a, 2^{k+1} r}, 2^{k+1} r \delta\right)$ respectively. Moreover, due to that $2^{J} r$ exceed the width of $A_{2}$, the two parts of $B_{2^{J} r}(a)-N\left(T_{a, 2^{J} r}, 2^{J} r \delta\right)$ meet $A_{1} \cap B_{3 / 4}$ and $A_{3} \cap B_{3 / 4}$ respectively. Note that if two connected subset meet, then they belong to the same connected component. Therefore, the two parts of $B_{r}(a)-N\left(T_{a, r}, r \delta\right)$ belong to $\Omega_{1}$ and $\Omega_{2}$ respectively. This proves (III).

(II) and (III) imply (IV).

Remark 4.2. Together, Theorems 4.1 and 1.4 imply Theorem 1.2 in the case $m=n-1$.

\section{Extension to parabolic space}

We now turn to the extension of the theorem of parabolic space. We start with some notations and definitions:

(1) $y=\left(y_{1}, \ldots, y_{n}\right)=\left(y^{\prime}, y_{n}\right)$ is a typical point of $\mathbb{R}^{n}$, and $y^{\prime} \in \mathbb{R}^{n-1}$. 
(2) $\left|y^{1}-y^{2}\right|=:\left(\sum_{i=1}^{n}\left(y_{i}^{1}-y_{i}^{2}\right)^{2}\right)^{1 / 2}$ and $\left\langle y^{1}, y^{2}\right\rangle=: \Sigma_{i=1}^{n} y_{i}^{1} y_{i}^{2}$ are the standard Euclidean metric and inner product.

(3) $x=(y, t) \in \mathbb{R}^{n} \times \mathbb{R}$ is a point of $\mathbb{R}^{n+1}$.

(4) The parabolic distance between $x^{1}$ and $x^{2}$ is defined as

$$
\left|x^{1}-x^{2}\right|_{p}:=\left(\left|y^{1}-y^{2}\right|^{2}+\left|t^{1}-t^{2}\right|\right)^{1 / 2},
$$

and $\mathbb{R}^{n+1}$ with this distance is called parabolic space.

(5) $B_{r}(a):=\left\{y \in \mathbb{R}^{n}:|y-a|<r\right\}$, where $a \in \mathbb{R}^{n}, B_{r}=: B_{r}(0)$.

(6) $T_{r}(a):=\left\{s \in \mathbb{R}^{n-1}:|s-a|<r\right\}$, where $a \in \mathbb{R}^{n-1}$.

(7) The parabolic ball is $\mathrm{p} B_{r}(a):=\left\{x \in \mathbb{R}^{n+1}:|x-a|_{p}<r\right\}$, where $a \in \mathbb{R}^{n+1}$.

(8) $p T_{r}(a):=\left\{z \in \mathbb{R}^{(n-1)+1}:|z-a|_{p}<r\right\}$, where $a \in \mathbb{R}^{(n-1)+1}$.

(9) A hyperplane in parabolic space $\mathbb{R}^{n+1}$ has the form $\tilde{E}=E \times \mathbb{R}$, namely, $\tilde{E}=:\left\{(y, t) \in \mathbb{R}^{n+1}: y \in E\right\}$, where $E$ is a hyperplane in Euclidean $\mathbb{R}^{n}$.

(10) For $y^{0} \in \mathbb{R}^{n}$ and $\vec{n} \in \mathbb{R}^{n}$ with $|\vec{n}|=1$, we denote by $E\left(y^{0}, \vec{n}\right):=\left\{y \in \mathbb{R}^{n}\right.$ : $\left.\left\langle y-y^{0}, \vec{n}\right\rangle=0\right\}$ the hyperplane passing through $y^{0}$ with normal vector $\vec{n}$ in $\mathbb{R}^{n}$.

In the codimension- 1 case, if we substitute parabolic ball, parabolic hyperplane and parabolic distance for Euclidean ball, hyperplane and distance in Definition 1.1, we get the definition of a Reifenberg flat set in parabolic space. We give two simple examples demonstrating what such set looks like. It is easy to verify them.

Example 5.1. In parabolic $\mathbb{R} \times \mathbb{R}$, the line with slope $k$, namely, the graph of $t=k y+b$ is $(\delta, R)$-Reifenberg flat if $R<\frac{1}{2} \delta k$.

Example 5.2. In parabolic $\mathbb{R} \times \mathbb{R}$, the graph of $t=\operatorname{sign}(y) k|y|^{1+\alpha}$ is $(\delta, R)$ Reifenberg flat if $R<\left(k(\delta / 2)^{1+\alpha}\right)^{1 /(1-\alpha)}$, where $0 \leq \alpha<1$.

Now we state our second main theorem.

Theorem 5.3. Let $\Omega \subset \mathbb{R}^{n+1}$ be a domain such that the origin $O=(0,0)$ lies in $\partial \Omega$, with normal vector $\vec{n}(O, 2))=e_{n}$. Let $\delta>0$ be a (small) constant. Assume that, for every $x^{1}=\left(y^{1}, t^{1}\right) \in \mathrm{p} B_{2} \cap \partial \Omega$ and every $r>0$ satisfying $\mathrm{p} B_{r}\left(x^{1}\right) \subset \mathrm{p} B_{2}$, there exists a unit vector $\vec{n}\left(x^{1}, r\right) \in \mathbb{R}^{n}$ such that

$$
\begin{aligned}
\mathrm{p} B_{r}\left(x^{1}\right) \cap\left\{(y, t) \in \mathbb{R}^{n+1}\right. & \left.:\left\langle y-\left(y^{1}-r \delta \vec{n}\left(x^{1}, r\right)\right), \vec{n}\left(x^{1}, r\right)\right\rangle<0\right\} \\
& \subset \mathrm{p} B_{r}\left(x^{1}\right) \cap \Omega \\
& \subset\left\{(y, t) \in \mathbb{R}^{n+1}:\left\langle y-\left(y^{1}+r \delta \vec{n}\left(x^{1}, r\right)\right), \vec{n}\left(x^{1}, r\right)\right\rangle<0\right\} .
\end{aligned}
$$

Then there exist a map

$$
f: p T_{1 / 2} \subset \mathbb{R}^{(n-1)+1} \rightarrow \partial \Omega \cap \mathrm{p} B_{1}
$$


and numbers $0<\alpha<1$ and $\beta>1$ such that

$$
f\left(p T_{1 / 2}\right) \supset \partial \Omega \cap \mathrm{p} B_{1 / 4}
$$

and

$$
c_{2}\left|x^{1}-x^{2}\right|_{p}^{\beta} \leq\left|f\left(x^{1}\right)-f\left(x^{2}\right)\right|_{p} \leq c_{1}\left|x^{1}-x^{2}\right|_{p}^{\alpha}
$$

for any $x^{1}, x^{2} \in p T_{1 / 2}$.

Domains satisfying the "for every..." condition of Theorem 5.3 are called parabolic Reifenberg flat. Such domains are geometrically invariant with respect to parabolic scaling. For the study of parabolic equations on parabolic Reifenberg flat domains, see [Byun and Wang 2005].

The proof of Theorem 5.3 parallels that of Theorem 1.4, so we omit most details. Attention should be paid to the degenerate direction $t$.

The parabolic mollifier. We define

$$
\eta(y, t):= \begin{cases}C_{1}\left(\frac{1}{2}-|y|^{4}-|t|^{2}\right)^{3} & \text { if }|y|^{4}+|t|^{2} \leq \frac{1}{2} \\ 0 & \text { else }\end{cases}
$$

where the constant $C_{1}$ is so adjusted that $\int_{\mathbb{R}^{n+1}} \eta(y, t) d y d t=1$. Next define

$$
\eta_{\varepsilon}(y, t):=\varepsilon^{-(n+2)} \eta\left(\varepsilon^{-1} y, \varepsilon^{-2} t\right)
$$

Thus

$$
\eta_{\varepsilon}(y, t) \in C_{0}^{2}\left(\mathrm{p} B_{\varepsilon}\right)
$$

and

$$
\int_{\mathbb{R}^{n+1}} \eta_{\varepsilon}(y, t) d y d t=1 .
$$

Level sets. From now on, we assume $\Omega$ is as in Theorem 5.3. Let $\chi_{\Omega}$ be the characteristic function of $\Omega$, and define

$$
\begin{aligned}
\chi_{\varepsilon} & :=\chi_{\Omega} * \eta_{\varepsilon}, \\
L_{\varepsilon} & :=\left\{x \in \mathbb{R}^{n+1}: \chi_{\varepsilon}(x)=\frac{1}{2}\right\}, \quad \Omega_{t}:=\left\{y \in \mathbb{R}^{n},(y, t) \in \Omega\right\}, \\
L_{\varepsilon, t} & :=\left\{y \in \mathbb{R}^{n}:(y, t) \in L_{\varepsilon}\right\}, \quad \partial \Omega_{t}:=\left\{y \in \mathbb{R}^{n},(y, t) \in \partial \Omega\right\} .
\end{aligned}
$$

Curvature estimate of the $\frac{\mathbf{1}}{\mathbf{2}}$-level set $\boldsymbol{L}_{\boldsymbol{\varepsilon}, \boldsymbol{t}}$. Fix $y^{0} \in L_{\varepsilon}, t^{0}$, and let $y^{1} \in \partial \Omega_{t^{0}}$ be such that $\left|y^{1}-y^{0}\right|=\inf _{y \in \partial \Omega_{t} 0}\left|y-y^{0}\right|=: d$. Obviously, $d<\varepsilon$. Define

$$
\begin{aligned}
A_{1}: & =\left\{(y, t):\left\langle y-\left(y^{1}-4 \varepsilon \delta \vec{n}\left(\left(y^{1}, t^{0}\right), 4 \varepsilon\right)\right), \vec{n}\left(\left(y^{1}, t^{0}\right), 4 \varepsilon\right)\right\rangle<0\right\} \cap \mathrm{p} B_{4 \varepsilon}\left(y^{1}, t^{0}\right), \\
A_{2} & :=\left\{(y, t):\left\langle y-\left(y^{1}+4 \varepsilon \delta \vec{n}\left(\left(y^{1}, t^{0}\right), 4 \varepsilon\right)\right), \vec{n}\left(\left(y^{1}, t^{0}\right), 4 \varepsilon\right)\right\rangle \geq 0\right\} \cap \mathrm{p} B_{4 \varepsilon}\left(y^{1}, t^{0}\right) \\
A_{3} & :=\mathrm{p} B_{4 \varepsilon}\left(y^{1}, t^{0}\right)-\left(A_{1} \cup A_{2}\right), \\
T & :=\partial A_{1}-\partial \mathrm{p} B_{4 \varepsilon}\left(y^{1}, t^{0}\right) .
\end{aligned}
$$


Lemma 5.4. $\left(y^{0}, t^{0}\right) \in A_{2}$, and $d \leq 8 \varepsilon \delta$.

Proof. The proof is the same as for Lemma 2.1.

We assume that $e_{n}=-\vec{n}\left(\left(y^{1}, t^{0}\right), 4 \varepsilon\right)$. Let $\hat{e}$ be any unit vector in $\mathbb{R}^{n-1}$ and set $e:=(\hat{e}, 0) \in \mathbb{R}^{n}$.

Lemma 5.5. For any $\bar{x}=(\bar{y}, \bar{t}) \in A_{2} \cap \mathrm{p} B_{\varepsilon}\left(y^{1}, t^{0}\right)$ we have

$$
\begin{gathered}
\frac{\partial}{\partial e_{n}} \chi_{\varepsilon}(\bar{x}) \geq C_{2} \varepsilon^{-1}, \quad\left|\frac{\partial}{\partial e} \chi_{\varepsilon}(\bar{x})\right| \leq C_{3} \delta \varepsilon^{-1}, \quad\left|\frac{\partial}{\partial t} \chi_{\varepsilon}(\bar{x})\right| \leq C_{4} \delta \varepsilon^{-2}, \\
\left|\frac{\partial^{2}}{\partial e^{2}} \chi_{\varepsilon}(\bar{x})\right| \leq C_{5} \delta \varepsilon^{-2}, \quad\left|\frac{\partial^{2}}{\partial e \partial e_{n}} \chi_{\varepsilon}(\bar{x})\right| \leq C_{5} \delta \varepsilon^{-2}, \quad\left|\frac{\partial^{2}}{\partial e_{n}^{2}} \chi_{\varepsilon}(\bar{x})\right| \leq C_{6} \delta \varepsilon^{-2}, \\
\left|\frac{\partial^{2}}{\partial e \partial t} \chi_{\varepsilon}(\bar{x})\right| \leq C_{7} \delta \varepsilon^{-3}\left|\frac{\partial^{2}}{\partial e_{n} \partial t} \chi_{\varepsilon}(\bar{x})\right| \leq C_{8} \delta \varepsilon^{-3} .
\end{gathered}
$$

Proof of the third inequality.

$$
\begin{aligned}
\frac{\partial}{\partial t} \chi_{\varepsilon}(\bar{x}) & =\int_{\Omega} \frac{\partial}{\partial t} \eta_{\varepsilon}(\bar{y}-y, \bar{t}-t) d y d t \\
& =\int_{A_{1} \cap \mathrm{p} B_{\varepsilon}(\bar{y}, \bar{t})} \frac{\partial}{\partial t} \eta_{\varepsilon}(\bar{y}-y, \bar{t}-t) d y d t \\
& \quad+\int_{\Omega \cap A_{2} \cap \mathrm{p} B_{\varepsilon}(\bar{y}, \bar{t})} \frac{\partial}{\partial t} \eta_{\varepsilon}(\bar{y}-y, \bar{t}-t) d y d t \\
& =: I_{5}+I_{6} ;
\end{aligned}
$$

but $I_{5}=0$ by Green's formula, and

$$
\begin{aligned}
\left|I_{6}\right| & \leq \int_{A_{2} \cap \mathrm{p} B_{\varepsilon}(\bar{y}, \bar{t})}\left|\frac{\partial}{\partial t} \eta_{\varepsilon}(\bar{y}-y, \bar{t}-t)\right| d y d t \\
& \leq C_{4}^{\prime} \varepsilon^{-(n+2)} \varepsilon^{-2} \cdot C_{4}^{\prime \prime} \delta \varepsilon^{n+2}=C_{4} \delta \varepsilon^{-2} .
\end{aligned}
$$

From the preceding lemma, we know that $\nabla \chi_{\varepsilon}(x) \neq 0$ for $x \in L_{\varepsilon} \cap \mathrm{p} B_{1}$; thus $L_{\varepsilon} \cap \mathrm{p} B_{1}$ is a $C^{2} n$-dimensional manifold and can be represent as the graph of some function from $\mathbb{R}^{n-1} \times \mathbb{R}$ to $\mathbb{R}$ locally. Take $x \in L_{\varepsilon} \cap \mathrm{p} B_{1}$ of the form $\left(y^{0}, t^{0}\right)$; then in $A_{2} \cap \mathrm{p} B_{\varepsilon}\left(y^{1}, t^{0}\right)$, the function can be taken as

$$
y_{n}=\varphi\left(y_{1}, \ldots, y_{n-1}, t\right)=: \varphi\left(y_{T}, t\right) .
$$

\section{Lemma 5.6.}

$$
\begin{aligned}
& \left|\frac{\partial}{\partial \hat{e}} \varphi\left(y_{T}, t\right)\right|<\frac{C_{3}}{C_{2}} \delta, \quad\left|\frac{\partial^{2}}{\partial \hat{e}^{2}} \varphi\left(y_{T}, t\right)\right| \leq C_{9} \delta \varepsilon^{-1}, \\
& \left|\frac{\partial}{\partial t} \varphi\left(y_{T}, t\right)\right|<\frac{C_{4}}{C_{2}} \delta \varepsilon^{-1},\left|\frac{\partial^{2}}{\partial \hat{e} \partial t} \varphi\left(y_{T}, t\right)\right| \leq C_{10} \delta \varepsilon^{-2} .
\end{aligned}
$$

Proof. The proof is the same as for Lemma 2.3. 
Corollary 5.7. For any $t \in(-1,1), L_{\varepsilon, t} \cap B_{1}$ is a $C^{2}(n-1)$-dimensional manifold in $\mathbb{R}^{n}$. The principal curvature of $L_{\varepsilon, t}$ in any tangent direction is less than $C_{9} \delta \varepsilon^{-1}$. So a tubular neighborhood with radii $\varepsilon$ exists as long as $C_{9} \delta<1$.

Closeness of $\boldsymbol{L}_{\boldsymbol{\varepsilon}}$ to $\partial \boldsymbol{\Omega}$. Recall from page 326 the notation $N(E, s)$ for an $s$-tubular neighborhood of $E$ and $\pi$ for the corresponding normal projection map. Further, for $F \in \mathbb{R}^{n+1}$, let $F_{t}=:\left\{y \in \mathbb{R}^{n}:(y, t) \in F\right\}$. If each $F_{t}$ has a tubular neighborhood of radius $\tau$, we define the parabolic tubular neighborhood

$$
\mathrm{p} N(F, \tau):=\bigcup_{t} N\left(F_{t}, \tau\right) \times\{t\},
$$

and the corresponding projection map $\tilde{\pi}: \mathrm{p} N(F, \tau) \rightarrow F$ such that $\tilde{\pi}(y, t)=$ $\left(\pi_{t} y, t\right)$, where $\pi_{t}$ is the normal projection operator of $N\left(F_{t}, \tau\right)$.

Lemma 5.8. $\partial \Omega \cap \mathrm{p} B_{r-\varepsilon} \subset \mathrm{p} N\left(L_{\varepsilon} \cap \mathrm{p} B_{r}, 4 \varepsilon \delta\right)$, for $r \leq 1$.

Proof. Same as for Lemma 2.5.

Taking $\varepsilon=4^{-k}, k=1,2, \ldots$, we obtain a sequence of approximate sets $L_{4^{-k}}$. Denote $\mathscr{Y}_{0}=:\left\{y_{n}=0\right\} \cap \mathrm{p} B_{1 / 2}, \mathscr{Y}_{k}=: L_{4^{-k}} \cap \mathrm{p} B_{r(k)}$, where $r(k)=\frac{1}{2}+\sum_{j=1}^{k} 4^{-j}$.

Corollary 5.9. $\mathscr{S}_{k} \in \mathrm{p} N\left(L_{4^{-k+1}}, 6 \cdot 4^{-k+1} \delta\right)$.

Proof. Same as for Corollary 2.6.

\section{Bi-Lipschitz maps.}

Lemma 5.10. $\tilde{\pi}: \mathscr{Y}_{k} \rightarrow L_{4^{-k+1}}$ is injective, and if $\delta$ is small enough, $\left|z^{1}-z^{2}\right|_{p} \leq$ $4^{-k}$, then

$$
\frac{1}{2}\left|\tilde{\pi}\left(z^{1}\right)-\tilde{\pi}\left(z^{2}\right)\right|_{p} \leq\left|z^{1}-z^{2}\right|_{p} \leq 2\left|\tilde{\pi}\left(z^{1}\right)-\tilde{\pi}\left(z^{2}\right)\right|_{p} .
$$

Proof. We write $x^{1}=: \tilde{\pi}\left(z^{1}\right)=\left(y^{1}, t^{1}\right), x^{2}=: \tilde{\pi}\left(z^{2}\right)=\left(y^{2}, t^{2}\right) \in L_{4^{-k+1}}$, and $z^{1}=\left(y^{3}, t^{1}\right), z^{2}=\left(y^{4}, t^{2}\right) \in \mathscr{Y}_{k}$. For simplicity, we still write $4^{-k+1}$ as $\varepsilon$ sometimes. We assume that $O$ is the nearest point of $\partial \Omega \cap\left\{t=t^{1}\right\}$ to $z^{1}$, by choosing a appropriate coordinate system, we can let $O=(0,0)$ (that means $\left.t^{1}=0\right)$ and $\vec{n}(0,4 \varepsilon)=-e_{n}$. Define

$$
\begin{aligned}
& A_{1}:=\left\{(y, t): y_{n}>4 \varepsilon \delta\right\} \cap \mathrm{p} B_{4 \varepsilon} \\
& A_{2}:=\left\{(y, t):-4 \varepsilon \delta \leq y_{n} \leq 4 \varepsilon \delta\right\} \cap \mathrm{p} B_{4 \varepsilon}, \\
& A_{3}:=\left\{(y, t): y_{n}<-4 \varepsilon \delta\right\} \cap \mathrm{p} B_{4 \varepsilon} .
\end{aligned}
$$

Then $A_{1} \subset \Omega, A_{3} \subset \bar{\Omega}^{c}$, and $x^{1}, x^{2}, z^{1}, z^{2} \in A_{2} \cap \mathrm{p} B_{\varepsilon}$.

We assume $L_{\varepsilon} \cap \mathrm{p} B_{\varepsilon}$ is the graph of a function $y_{n}=\varphi\left(y_{T}, t\right)=\varphi\left(y_{1}, \ldots, y_{n-1}, t\right)$, and $L_{\varepsilon / 4} \cap \mathrm{p} B_{\varepsilon}$ is the graph of a function $y_{n}=\psi\left(y_{T}, t\right)$. Thus, we can write

$$
\begin{aligned}
& x^{1}=\left(y_{T}^{1}, \varphi\left(y_{T}^{1}, 0\right), 0\right), \quad x^{2}=\left(y_{T}^{2}, \varphi\left(y_{T}^{2}, t^{2}\right), t^{2}\right), \\
& z^{1}=\left(y_{T}^{3}, \psi\left(y_{T}^{3}, 0\right), 0\right), \quad z^{2}=\left(y_{T}^{4}, \psi\left(y_{T}^{4}, t^{2}\right), t^{2}\right) .
\end{aligned}
$$


Set $l:=\left|y_{T}^{1}-y_{T}^{2}\right|$ and $\hat{l}:=\left|y_{T}^{3}-y_{T}^{4}\right|$; by assumption,

$$
\hat{l} \leq \varepsilon / 4, \quad l \leq \varepsilon / 2, \quad\left|t^{2}\right| \leq \varepsilon^{2} .
$$

By Lemma 5.6 and the Differential Mean Value Theorem,

$$
\begin{aligned}
& \left|\varphi\left(y_{T}^{1}, 0\right)-\varphi\left(y_{T}^{2}, t^{2}\right)\right| \leq l \frac{C_{3}}{C_{2}} \delta+\left|t^{2}\right| \frac{C_{4}}{C_{2}} \delta \varepsilon^{-1}, \\
& \left|\psi\left(y_{T}^{3}, 0\right)-\varphi\left(y_{T}^{4}, t^{2}\right)\right| \leq \hat{l} \frac{C_{3}}{C_{2}} \delta+\left|t^{2}\right| \frac{C_{4}}{C_{2}} \delta \varepsilon^{-1} .
\end{aligned}
$$

Then

$$
\begin{aligned}
\sqrt{l^{2}+\left|t^{2}\right|} \leq\left|x^{1}-x^{2}\right|_{p} & \leq \sqrt{l^{2}+2\left(\frac{C_{3}}{C_{2}} \delta\right)^{2} l^{2}+2\left(\frac{C_{4}}{C_{2}} \delta\right)^{2} \varepsilon^{-2}\left|t^{2}\right|^{2}+\left|t^{2}\right|} \\
& \leq \frac{11}{10} \sqrt{l^{2}+\left|t^{2}\right|} \quad \text { (using } \delta \text { small and (5-1)) }
\end{aligned}
$$

and

$$
\sqrt{\hat{l}^{2}+\left|t^{2}\right|} \leq\left|z^{1}-z^{2}\right|_{p} \leq \frac{11}{10} \sqrt{\hat{l}^{2}+\left|t^{2}\right|} .
$$

Since $\tilde{\pi}\left(z^{1}\right)=x^{1}, \tilde{\pi}\left(z^{2}\right)=x^{2}$, there exist $\xi$ and $\zeta \in \mathbb{R}$ such that

$$
y^{3}=y^{1}+\xi v\left(x^{1}\right), \quad y^{4}=y^{2}+\zeta v\left(x^{2}\right),
$$

where $v\left(x^{1}\right), v\left(x^{2}\right)$ are inward-pointing unit normal vectors of $L_{\varepsilon}$ at $x^{1}, x^{2}$ (with expressions similar to those near the bottom of page 327). By Corollary 5.9,

$$
|\xi|,|\zeta| \leq 6 \cdot \varepsilon \delta
$$

moreover, $\left|v_{i}\left(x^{j}\right)\right|<C_{3} / C_{2} \delta$ for $i=1, \ldots, n-1$, and, by Lemma 5.6,

$$
v_{n}\left(x^{j}\right)>\frac{1}{\sqrt{1+\left(C_{3} / C_{2} \delta\right)^{2}}} \text { for } j=1,2 .
$$

These two inequalities show that $v\left(x^{j}\right)$ is very close to $e_{n}$ when $\delta$ is very small.

Subtracting the second equality in (5-5) from the first, we get

$$
\begin{aligned}
& y_{i}^{3}-y_{i}^{4}=y_{i}^{1}-y_{i}^{2}+\xi\left(v_{i}\left(x^{1}\right)-v_{i}\left(x^{2}\right)\right)+(\xi-\zeta) v_{i}\left(x^{2}\right), \\
& y_{n}^{3}-y_{n}^{4}=y_{n}^{1}-y_{n}^{2}+\xi\left(v_{n}\left(x^{1}\right)-v_{n}\left(x^{2}\right)\right)+(\xi-\zeta) v_{n}\left(x^{2}\right) .
\end{aligned}
$$

By Lemma 5.6 and the Differential Mean Value Theorem,

$$
\begin{aligned}
& \left|v_{i}\left(x^{1}\right)-v_{i}\left(x^{2}\right)\right| \\
& \quad \leq\left|D_{i} \varphi\left(y_{T}^{2}, t^{2}\right)-D_{i} \varphi\left(y_{T}^{1}, 0\right)\right|+\left|\nabla_{y_{T}} \varphi\left(y_{T}^{1}, 0\right)\right|^{2} \cdot\left|\nabla_{y_{T}} \varphi\left(y_{T}^{2}, t^{2}\right)-\nabla_{y_{T}} \varphi\left(y_{T}^{1}, 0\right)\right| \\
& \quad \leq 2\left(l \cdot C_{9} \delta \varepsilon^{-1}+\left|t^{2}\right| \cdot C_{10} \delta \varepsilon^{-2}\right)
\end{aligned}
$$


and

$$
\begin{aligned}
\mid v_{n}\left(x^{1}\right) & -v_{n}\left(x^{2}\right) \mid \\
& \leq\left(\left|\nabla_{y_{T}} \varphi\left(y_{T}^{1}, 0\right)\right|+\left|\nabla_{y_{T}} \varphi\left(y_{T}^{2}, t^{2}\right)\right|\right) \cdot\left|\nabla_{y_{T}} \varphi\left(y_{T}^{1}, 0\right)-\nabla_{y_{T}} \varphi\left(y_{T}^{2}, t^{2}\right)\right| \\
& \leq l C_{9} \delta \varepsilon^{-1}+\left|t^{2}\right| C_{10} \delta \varepsilon^{-2} .
\end{aligned}
$$

Equality (5-8) implies

$$
\begin{aligned}
|\xi-\zeta| & \leq \sqrt{1+\left(\frac{C_{3}}{C_{2}} \delta\right)^{2}}\left(\left|y_{n}^{3}-y_{n}^{4}\right|+\left|y_{n}^{1}-y_{n}^{2}\right|+|\xi|\left|v_{n}\left(x^{1}\right)-v_{n}\left(x^{2}\right)\right|\right) \\
& \leq \sqrt{1+\left(\frac{C_{3}}{C_{2}} \delta\right)^{2}}\left(\hat{l} \frac{C_{3}}{C_{2}} \delta+\left|t^{2}\right| \frac{C_{4}}{C_{2}} \delta \varepsilon^{-1}+l \frac{C_{3}}{C_{2}} \delta+\left|t^{2}\right| \frac{C_{4}}{C_{2}} \delta \varepsilon^{-1}\right. \\
& \left.+5 \varepsilon \delta\left(l C_{9} \delta \varepsilon^{-1}+\left|t^{2}\right| C_{10} \delta \varepsilon^{-2}\right)\right) \\
& \leq C_{11}\left(\delta l+\delta \hat{l}+\delta \varepsilon^{-1}\left|t^{2}\right|\right),
\end{aligned}
$$

where we used (5-2), (5-6) and (5-9) in the second inequality and the third holds for $\delta$ small.

Using the triangle inequality, (5-7) implies

$$
\begin{aligned}
\hat{l} & \leq l+|\xi|\left|v_{i}\left(x^{1}\right)-v_{i}\left(x^{2}\right)\right|+|\xi-\zeta|\left|v_{i}\left(x^{2}\right)\right| \\
& \leq\left(1+C_{12} \delta^{2}\right) l+C_{11} \frac{C_{3}}{C_{2}} \delta^{2} \hat{l}+C_{12} \delta^{2} \varepsilon^{-1}\left|t^{2}\right| .
\end{aligned}
$$

Thus, if $\delta$ is small enough,

$$
\hat{l} \leq \frac{11}{10} l+\delta \varepsilon^{-1}\left|t^{2}\right|
$$

Similarly, we can show that

$$
\hat{l} \geq \frac{9}{10} l-\delta \varepsilon^{-1}\left|t^{2}\right|
$$

Therefore,

$$
\sqrt{\hat{l}^{2}+\left|t^{2}\right|} \leq \sqrt{2\left(\frac{11}{10}\right)^{2} l^{2}+2 \delta^{2} \varepsilon^{-2}\left|t^{2}\right|^{2}+\left|t^{2}\right|} \leq \sqrt{2} \frac{11}{10} \sqrt{l^{2}+\left|t^{2}\right|}
$$

where we have used (5-1). On the other hand, if $l^{2} \leq\left|t^{2}\right|$, we have

$$
\sqrt{\hat{l}^{2}+\left|t^{2}\right|} \geq \sqrt{\left|t^{2}\right|} \geq \frac{1}{\sqrt{2}} \sqrt{l^{2}+\left|t^{2}\right|}
$$

and if $l^{2}>\left|t^{2}\right|$, then (5-10) becomes

$$
\hat{l}>\frac{9}{10} l-\delta \varepsilon^{-1} l^{2}>\left(\frac{9}{10}-\delta\right) l>\frac{4}{5} l
$$


(using (3-1)), so we also have

$$
\sqrt{\hat{l}^{2}+\left|t^{2}\right|} \geq \frac{4}{5} \sqrt{l^{2}+\left|t^{2}\right|}
$$

Combining (5-3), (5-4), (5-11), (5-12) and (5-13), we conclude the proof.

We define $g_{k}: \mathscr{Y}_{k-1} \rightarrow \mathscr{Y}_{k}$ satisfying $\tilde{\pi}\left(g_{k}(x)\right)=x$ for $k=1,2, \ldots$, and $h_{k}$ : $\mathscr{I}_{0} \rightarrow \mathscr{Y}_{k}$ by setting $h_{k}:=g_{k} \circ \cdots \circ g_{1}$.

Corollary 5.11. $\left|h_{k}(x)-h_{k-1}(x)\right|=\left|g_{k}\left(h_{k-1}(x)\right)-h_{k-1}(x)\right|<6 \cdot 4^{-k+1} \delta$.

Proof of Theorem 5.3. The proof is assembled from Corollary 5.11 and Lemmas 5.8 and 5.10 in precisely the same way as the proof of Theorem 1.4 (Section 3), with obvious modifications to the notation. The same constants can be used.

\section{Acknowledgment}

The authors thank the referee for several useful comments.

\section{References}

[Byun and Wang 2004] S.-S. Byun and L. Wang, "Elliptic equations with BMO coefficients in Reifenberg domains", Comm. Pure Appl. Math. 57:10 (2004), 1283-1310. MR 2006b:35058 Zbl 02126546

[Byun and Wang 2005] S.-S. Byun and L. Wang, "Parabolic equations in Reifenberg domains", Arch. Ration. Mech. Anal. 176:2 (2005), 271-301. MR 2007h:35142 Zbl 1073.35046

[Capogna et al. 2005] L. Capogna, C. E. Kenig, and L. Lanzani, Harmonic measure: geometric and analytic points of view, University Lecture Series 35. American Mathematical Society, Providence, RI, 2005. MR 2006a:31002 Zbl 1074.31001

[David and Toro 1999] G. David and T. Toro, "Reifenberg flat metric spaces, snowballs, and embeddings", Math. Ann. 315:4 (1999), 641-710. MR 2001c:49067 Zbl 0944.53004

[David et al. 2001] G. David, C. Kenig, and T. Toro, "Asymptotically optimally doubling measures and Reifenberg flat sets with vanishing constant", Comm. Pure Appl. Math. 54:4 (2001), 385-449. MR 2002g:28007 Zbl 1031.28004

[Kenig and Toro 1997] C. E. Kenig and T. Toro, "Harmonic measure on locally flat domains", Duke Math. J. 87:3 (1997), 509-551. MR 98k:31010 Zbl 0878.31002

[Kenig and Toro 1999] C. E. Kenig and T. Toro, "Free boundary regularity for harmonic measures and Poisson kernels", Ann. of Math. (2) 150:2 (1999), 369-454. MR 2001d:31004 Zbl 0946.31001

[Lin and Yang 2002] F. Lin and X. Yang, Geometric measure theory: an introduction, Science Press, Beijing and International Press, Boston, 2002. MR 2005a:28001 Zbl 1074.49011

[Morrey 1966] C. B. Morrey, Jr., Multiple integrals in the calculus of variations, Grundlehren der mathematischen Wissenschaften 130, Springer, New York, 1966. MR 34 \#2380 Zbl 0142.38701

[Reifenberg 1960] E. R. Reifenberg, "Solution of the Plateau problem for $m$-dimensional surfaces of varying topological type”, Acta Math. 104 (1960), 1-92. MR 22 \#4972 Zbl 0099.08503

[Toro 1997] T. Toro, "Doubling and flatness: geometry of measures", Notices Amer. Math. Soc. 44:9 (1997), 1087-1094. MR 99d:28010 Zbl 0909.31006 
Received January 5, 2007. Revised May 31, 2007.

GuANGHAO HONG

East CAmpus, MaIlboX 1924

XI'AN JIAOTONG UNIVERSITY

XI'AN 710049

SHAANXI PROVINCE

CHINA

ghhongmath@gmail.com

LIHE WANG

DEPARTMENT OF MATHEMATICS

14 MaCLEAN HALL

UNIVERSITY OF IOWA

IOWA CITY, IA 52242-1419

UNITED STATES

lwang@math.uiowa.edu

www.math.uiowa.edu/ lwang 